\title{
Immunogenicity analysis following human immunodeficiency virus recombinant DNA and recombinant vaccinia virus Tian Tan prime-boost immunization
}

\author{
LIU CunXia ${ }^{1,2 \dagger}$, DU ShouWen ${ }^{1,2 \dagger}$, LI Chang $^{2 *}$, WANG YuHang ${ }^{2}$, WANG MaoPeng ${ }^{1,2}$, LI Yi $^{2}$, \\ YIN RongLan ${ }^{3}$, LI Xiao $^{2}$, REN DaYong ${ }^{1,2}$, QIN YanQing ${ }^{2}$, REN JingQiang ${ }^{1,2} \&$ JIN Ning Yi ${ }^{2 *}$ \\ ${ }^{1}$ College of Animal Science and Veterinary Medicine, Jilin University, Changchun 130062, China; \\ ${ }^{2}$ Key Laboratory of Jilin Province for Zoonosis Prevention and Control, Institute of Military Veterinary, AMMS, Changchun 130122, China; \\ ${ }^{3}$ Academy of Animal Science and Veterinary Medicine in Jilin Province, Changchun 130062, China
}

Received March 12, 2013; accepted April 13, 2013; published online May 3, 2013

\begin{abstract}
This study assessed and compared the immunogenicity of various immunization strategies in mice using combinations of recombinant DNA (pCCMp24) and recombinant attenuated vaccinia virus Tian Tan (rddVTT-CCMp24). Intramuscular immunization was performed on days 0 (prime) and 21 (boost). The immunogenicity of the vaccine schedules was determined by measuring human immunodeficiency virus (HIV)-specific binding antibody levels and cytokine (interleukin-2 and interleukin-4) concentrations in peripheral blood, analyzing lymphocyte proliferation capacity against HIV epitopes and $\mathrm{CD} 4^{+} / \mathrm{CD}^{+}$cell ratio, and monitoring interferon-gamma levels at different times post-immunization. The results showed that pCCMp24,

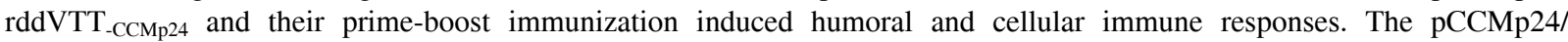
rddVTT $_{- \text {CCMp24 }}$ immunization strategy increased $\mathrm{CD}^{+} \mathrm{T}$ cells and induced more IFN- $\gamma$-secreting cells compared with single-shot rDNA. The prime-boost immunization strategy also induced the generation of cellular immunological memory to HIV

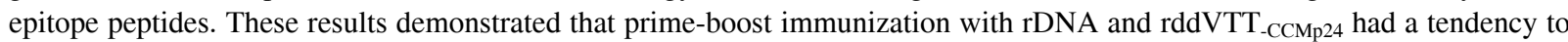
induce greater cellular immune response than single-shot vaccinations, especially IFN- $\gamma$ response, providing a basis for further studies.
\end{abstract}

vaccinia virus Tian Tan, human immunodeficiency virus, recombinant DNA, prime-boost, immunogenicity

Citation: Liu C X, Du S W, Li C, et al. Immunogenicity analysis following human immunodeficiency virus recombinant DNA and recombinant vaccinia virus Tian Tan prime-boost immunization. Sci China Life Sci, 2013, 56: 531-540, doi: 10.1007/s11427-013-4484-2

Acquired immunodeficiency syndrome (AIDS) is an epidemic disease that is difficult to both prevent and treat. Designing and developing of an effective preventive or therapeutic AIDS vaccine has been a great challenge to public health and scientific research. Recent studies in AIDS vaccine development have involved using (i) vaccine vectors, such as modified vaccinia Ankara (MVA) [1,2], New York

$\dagger$ Contributed equally to this work

*Corresponding author (email: ningyij@hotmail.com; lichang78@163.com) vaccinia (NYVAC) [3], fowlpox virus (FPV) [4,5], and Ad5 [6,7], (ii) various human immunodeficiency virus (HIV) immunogens, such as gp120 [8], env [9], tat [10], HIV multiple epitopes [11-13], and multi-genes [14,15], or (iii) different immunization routes and strategies [16,17]. Since the first AIDS vaccine trial in 1987, more than 230 vaccine trials have been conducted [18]. Unfortunately, most of these vaccines failed to induce protection against HIV infection [19]. In a recent phase III clinical trial (RV144) in Thailand, using ALVAC-HIV (a recombinant canarypox 
vector that expresses $g a g$, pol, and env) as prime and AIDSVAX B/E (a recombinant gp120 protein) as boost has shown $31.2 \%$ protective efficacy against HIV. This study has provided the first proof-of-concept for a successful HIV vaccine [20].

Recent studies have focused on developing novel DNAbased AIDS vaccines. However, to data, these vaccines have failed to confer protective efficacy in non-human primates and phase I/II human trials. To improve the immunogenicity of DNA-based vaccines, many new delivery methods and immunization strategies are being tested, including electroporation [21], repetitive vaccine injections [22], co-expression of cytokines, such as interleukin-12 (IL12) [23], 4-1BBL [24], or granulocyte-macrophage colonystimulating factor (GM-CSF) [25] as molecular adjuvants, and prime-boost immunization strategies using heterologous vaccines which encode similar antigens [26]. CpG oligodeoxynucleotides (ODN) are powerful immunomodulatory agents capable of enhancing innate immune responses and when used as an adjuvant to improve adaptive immune responses against pathogens [27-29]. Cholera toxin (CT) has the ability to prime memory CD8 T cells, which control bacterial and viral challenges [30]. In this study, CpG ODN and CT subunit B (CTB) served as adjuvants for DNA and recombinant viral vaccines.

Vaccinia virus-based vaccines expressing HIV-associated immunogens can induce both humoral and cellular immune responses [31]. In a phase I clinical trial, the recombinant virus rMVA expressing HIV-1 virus-like particles elicited different patterns of $\mathrm{T}$ cell and antibody responses when used alone or in combination with DNA vaccine [17]. Several studies have also shown that immune responses can be generated in vivo in small animals or non-human primates after boost immunization with recombinant poxvirus following rDNA [26]. This prime-boost strategy using HIV rDNA for priming and recombinant vaccinia virus for boosting has been pursued by many researchers. Studies in non-human primate models using the SHIV or SIV DNA vaccine for priming and recombinant MVA for boosting have demonstrated that this regimen helped reduce viral loads in vivo and controlled disease progression [32-34]. In healthy adults, regimens using a multiclade, multigene HIV-1 DNA vaccine for priming and MVA for boosting were considered safe and induced broad and potent immune responses [35,36]. Recent research has shown that rDNA and rMVA vaccines encoding multiple cytotoxic and helper T-lymphocyte epitopes of HIV-1 were weakly immunogenic but safe in HIV-1-uninfected, vaccinia virus-naive adults [37].

In the current study, rDNA and attenuated vaccinia virus Tian Tan (VTT) vector vaccines were constructed using the antigen MEGNp24 developed previously $[5,12,38]$. Two important molecular adjuvants, $\mathrm{CpG}$ oligodeoxynucleotides $(\mathrm{ODN})$ and cholera toxin subunit $\mathrm{B}(\mathrm{CTB})$, were combined with the vaccines to further enhance immunogenicity. Immunogenicity was evaluated in vitro and in vivo in cell models and in mice administered with individual vaccines or in combination using the prime-boost regimen. Antigenspecific humoral and cellular immune responses were analyzed for each vaccine regimen.

\section{Materials and methods}

\section{1 rDNA and vaccinia virus vaccines}

In the current study, rDNA pVL-CCMp24 (pCCMp24) and recombinant attenuated vaccinia virus Tian Tan $\left(\right.$ rddVTT $\left._{-\mathrm{CCMp} 24}\right)$ were prepared as described previously $(\mathrm{Du}$ et al., data not shown). The vaccines expressed 29 HIV dominant epitopes (24 CTL or CD8 T-cell epitopes and five B-cell epitopes), HIV-1 p24 protein, CpG ODN (5'AAAAACGTTGACGTTAACGTTGACGTT-3'), and CTB.


is shown in Figure 1A. The vaccines were diluted to the required concentrations in phosphate-buffered saline (PBS, pH 7.4).

\subsection{Immunization of mice}

Six-week-old female BALB/c mice were purchased from the Laboratory Animal Center, Academy of Military Medical Sciences (Beijing, China). All animal experimental protocols were approved by the Institutional Animal Care and Use Committee of the Chinese Academy of Military Medical Science (10ZDGG007). The mice were divided into four groups of eight for immunization. Group 1 was injected intramuscularly (i.m.) with $100 \mu \mathrm{g}$ pCCMp24, group 2 was injected i.m. with $100 \mu \mathrm{L}$ of $1 \times 10^{6} \mathrm{PFU} \mathrm{mL}^{-1}$

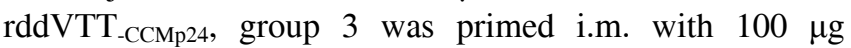
pCCMp24 and boosted with $100 \mu \mathrm{L}$ of $1 \times 10^{6} \mathrm{PFU} \mathrm{mL}^{-1}$

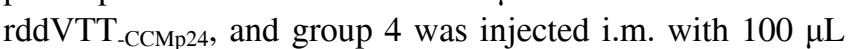
PBS. Mice from each group were boosted three weeks after the prime vaccination using the same inoculums as for priming unless stated otherwise. The immunization schemes are shown in Figure 1B.

\subsection{Preparation of splenocytes}

The splenocytes of immunized mice were isolated by centrifugation with Ficoll-Hypaque lymphocyte isolation solution (TBD Science, China) according to the manufacturer's instructions. In brief, at $10 \mathrm{~d}$ post-booster immunization, red blood cells were treated with lysis buffer and washed twice with complete medium, and the splenocytes were suspended in complete culture medium (89\% RPMI 1640; HyClone, China), $10 \%$ fetal bovine serum (HyClone), and $1 \%$ penicillin-streptomycin (HyClone). The splenocytes were then used for $\mathrm{T}$ cell measurements. 


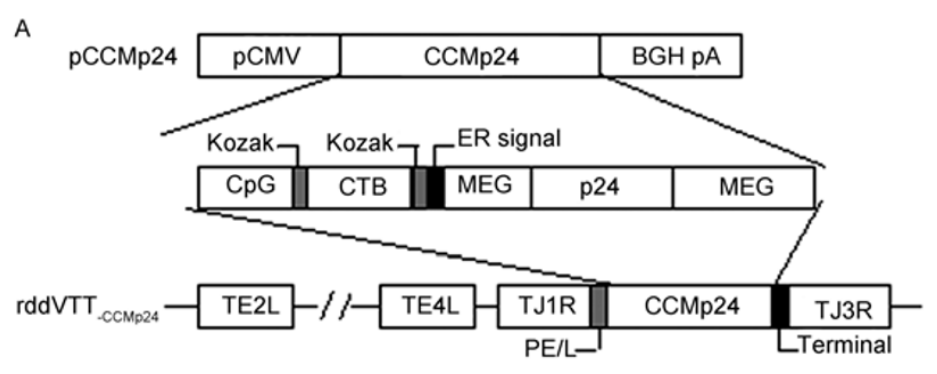

B
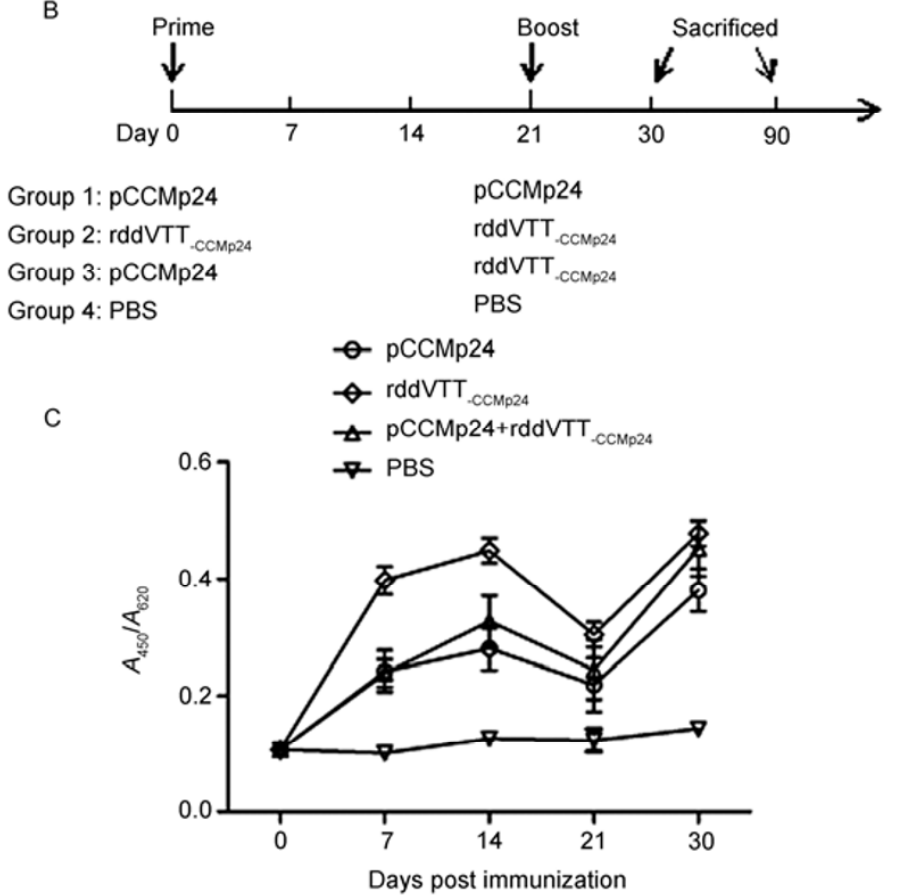

Figure 1 Schematic of pCCMp24 and rddVTT-CCMp24 construction (A) and immunization (B) and HIV specific antibody detection by ELISA (C). A, The fusion gene CCMp24 containing CpG ODN, CTB, and the multi-epitope gene (MEG), the 29 epitopes and HIV-1 p24 were inserted into the eukaryotic expression vector pVAX1 and expressed under the control of the human cytomegalovirus (CMV) promoter. CCMp24 was also introduced into the TJ2R (expressing thymidine kinase)-site of the genome of TE3L-deleted vaccinia virus Tian Tan (VTT) and expressed under vaccinia virus promoter PE/L. B, Mice were divided into four groups and vaccinated twice by intramuscular injection. C, ELISA-binding HIV-specific antibody responses elicited with different vaccines and different strategies. The serum samples from each group were collected at 7, 14, 21, and 30 d after vaccination and pre-immunization to ascertain anti-HIV IgG by ELISA. The total anti-HIV serum IgG at different periods is illustrated above. All data represent the mean ( $n=6) \pm$ SD.

\subsection{Collection of serum samples}

Blood samples were collected at 7, 14, 21, and $30 \mathrm{~d}$ following the initial vaccination (prime) and stored at $4{ }^{\circ} \mathrm{C}$ for $1 \mathrm{~h}$. The blood samples were centrifuged at $1000 \times \mathrm{g}$ for $10 \mathrm{~min}$, the sera were recovered and stored at $-70^{\circ} \mathrm{C}$ until required for further testing. Sera from pre-immune mice were separated and included as the negative control.

\subsection{HIV-specific antibody enzyme-linked immuno- sorbent assay (ELISA)}

The HIV antibody detection kit (Beijing Wantai Biological Pharmacy Enterprise Co., Ltd., Beijing, China) was used to measure HIV-1-specific antibody titers. Briefly, 96-well ELISA plates were coated with highly purified recombinant HIV-1 antigens. One hundred microliter serum samples (test samples, as well as positive and negative control sera) were added to the appropriate wells, and the plates incubated at $37^{\circ} \mathrm{C}$ for $1 \mathrm{~h}$ in a humid environment. The plates were washed five times with $1 \times$ washing buffer and $100 \mu \mathrm{L}$ HRP-conjugate reagent was added. Complex formation was permitted to proceed for $30 \mathrm{~min}$ at $37^{\circ} \mathrm{C}$ in a humid environment. The plates were washed five times, and $50 \mu \mathrm{L}$ chromogen solutions A and B added to each well. Color formation was performed for approximately $30 \mathrm{~min}$ in the absence of light. Stop solution $(50 \mu \mathrm{L} /$ well $)$ was added to each well to conclude the reaction. The absorbance values for each well were read at $450 \mathrm{~nm}$ using a microplate reader.

\subsection{Interleukin-2 (IL-2) and interleukin-4 (IL-4) ELISA assay}

The concentrations of IL-2 and IL-4 present in the peripheral blood of mice following booster immunization was measured using serum samples harvested at $10 \mathrm{~d}$ post- 
booster immunization using mouse IL-2 and IL-4 ELISA kits (GBD, USA) according to the manufacturer's instructions.

\subsection{Lymphocyte proliferative assay}

To assess the proliferative response of $\mathrm{T}$ lymphocytes against HIV-specific peptides, splenocytes were seeded in 96-well tissue culture plate at a cell density of $2.5 \times 10^{5}$ cells/well and co-cultured with HIV-1 peptide pools (gp160, VTVYYGVPVWK; Pol, VTIKIGGQLK; Rt, ILKEPVHGV; IN, LLWKGEGAV; Vpr, FPRPWLHSL; Tat, RKKRRQRRS; Nef, FPVRPQVPL, at $10 \mathrm{mg} \mathrm{L}^{-1}$ per peptide) as a specific antigenic stimulator and mitogen ConA as a positive control, for $72 \mathrm{~h}$ at $37^{\circ} \mathrm{C}$ in the presence of $5 \% \mathrm{CO}_{2}$. Splenocytes cultured with medium alone (no stimulation) served as a negative control. Twenty microliters of WST-1 solution (Beyotime Institute of Biotechnology, Haimen, China) was added to each well and incubated for $4 \mathrm{~h}$, prior to agitation for $1 \mathrm{~min}$. The absorbance values were measured at $450 \mathrm{~nm}$ using an ELISA microplate reader. The proliferation of splenocytes was represented by the stimulation index $(S I)$ : $S I=$ mean absorbance value $A_{450}$ of stimulated cells/mean absorbance value $A_{450}$ of negative control.

\section{8 $\mathrm{CD4}^{+}$and $\mathrm{CD8}^{+} \mathrm{T}$-cell subtype assay}

About $1.0 \times 10^{6}$ splenocytes sampled from mice at $10 \mathrm{~d}$ post-booster immunization were washed with FACS buffer and then stained with PerCP/Cy5.5-labeled anti-mouse CD3, fluorescein isothiocyanate-labeled anti-mouse CD4, and phycoerythrin-labeled anti-mouse CD8 (BioLegend, CA, USA) at $4{ }^{\circ} \mathrm{C}$ in the dark for $30 \mathrm{~min}$. The samples were washed twice, fixed in PBS containing $1 \%$ paraformaldehyde and analyzed by flow cytometry using Coulter Epics XL (Beckman Coulter, USA). Statistical analysis of the percentage of $\mathrm{CD}^{+} \mathrm{CD}^{+}$and $\mathrm{CD}^{+} \mathrm{CD}^{+} \mathrm{T}$ lymphocytes was performed.

\subsection{Interferon-gamma (IFN- $\gamma)$ enzyme-linked im- munosorbent spot (ELISPOT) assay}

Vaccine and HIV-specific cellular immune responses in mice were evaluated by ELISPOT assay measurements of splenocyte IFN- $\gamma$ secretion following stimulation with heat-inactivated recombinant vaccinia virus or HIV CTL epitope peptides. Briefly, $1.0 \times 10^{5}$ splenocytes were plated in triplicate in 96-well polyvinylidene difluoride membrane plates coated with captured antibody using a mouse IFN- $\gamma$ precoated ELISPOT kit according to the manufacturer's instructions (Dakewe Biotech, Shenzhen, China). The cells were stimulated with either $10 \mu \mathrm{L}$ heat-inactivated $\operatorname{rddVTT}_{-\mathrm{CCMp} 24}\left(1.0 \times 10^{6} \mathrm{PFU} \mathrm{mL} \mathrm{mL}^{-1}\right)$ or a pool of HIV epitope peptides (gp160, VTVYYGVPVWK; Pol, VTIKIGGQLK; Rt, ILKEPVHGV; IN, LLWKGEGAV; Vpr, FPRPWLHSL;
Tat, RKKRRQRRS; Nef, FPVRPQVPL, at $10 \mathrm{mg} \mathrm{L}^{-1}$ per peptide). Phytohemagglutinin $\left(2.5 \mu \mathrm{g} \mathrm{mL}^{-1}\right)$ was used as the positive control. RPMI 1640 was used as the negative control. After stimulation for $24 \mathrm{~h}$, the cells were washed, IFN- $\gamma$ secreting cells developed using biotinylated antibody and streptavidin-HRP, and the cells counted with a Biosys Bioreader.

\subsection{Statistical analysis}

Statistical analysis and comparisons between immunization groups was performed using Graphpad Prism software (San Diego, CA, USA). Data are presented as the mean \pm standard deviation (SD). The significance of differences was calculated using the One-way ANOVA (two tailed, confidence intervals 95\%), as indicated by the $P$-value. Differences with a $P$-value $<0.05$ were considered to be statistically significant.

\section{Results}

\subsection{HIV-specific antibody response induced by differ-} ent regimens

HIV-specific IgG present in the peripheral blood of mice immunized with the nominated vaccines and immunization strategies was measured at 7, 14, 21, and $30 \mathrm{~d}$ postimmunization by ELISA (Figure 1B). The results demonstrated consistent changes in IgG levels between the immunized groups and the PBS control group. As shown in Figure $1 \mathrm{C}$, at $14 \mathrm{~d}$ post-immunization, the total antibody levels increased and then decreased. Following booster administration at $21 \mathrm{~d}$, HIV-specific IgG levels again increased. At $7 \mathrm{~d}$ post-prime, the total antibody levels were higher for all vaccine groups analyzed when compared with the PBS control group $(P<0.05)$, and the antibody levels


were markedly higher than for pCCMp24 (Group 1) $(P<0.05$; Figure $1 C)$. For the rDNA/virus prime-boost immunization group (Group 3), at $10 \mathrm{~d}$ post-boost with

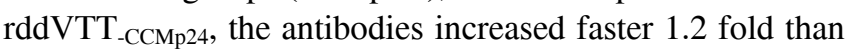
for pCCMp24-boost. The above results suggest that rddVTT $_{\text {-CCMp24 }}$ induced HIV-specific IgG responses, and it is obvious that the use of $\operatorname{rddVTT}_{-\mathrm{CCMp}_{24}}$ can enhance the immune response as a booster.

\subsection{IL-2 and IL-4 secretion induced by different regi- mens}

The concentration of cytokines IL-2 and IL-4 in the peripheral blood of mice was determined using a double antibody ELISA. As shown in Figure 2A and B, the concentrations of IL-2 and IL-4 were markedly higher for all immunization groups analyzed when compared with the PBS control 

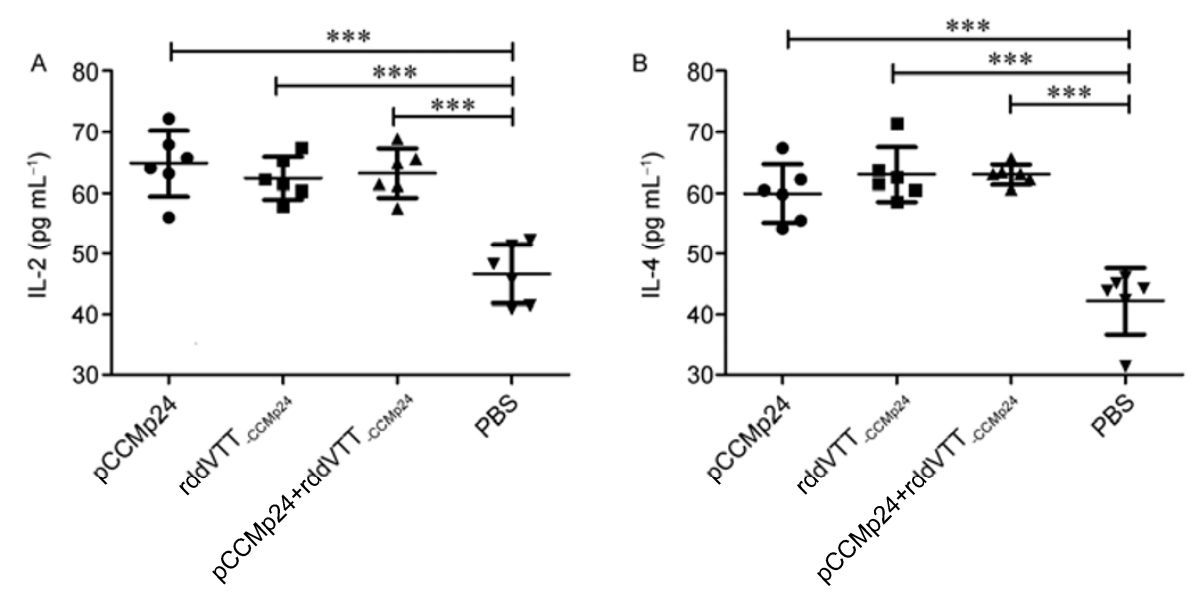

Figure 2 IL-2 and IL-4 levels in peripheral blood measured by ELISA. Three serum samples from each group were collected at $30 \mathrm{~d}$ after prime vaccination and used to determine IL-2 and IL-4 levels $\left(\mathrm{pg} \mathrm{mL}^{-1}\right)$. The data represent the mean $(n=6) \pm \mathrm{SD}$. Asterisks indicate statistically significant differences relative to the PBS control; ***, $P<0.001$.

group $(P<0.01)$. The results indicated that pCCMp24, rddVTT $_{-\mathrm{CCMp}_{2}}$ and their prime-boost regimen all induced the generation and secretion of IL-2 and IL-4.

\subsection{Splenic T-lymphocyte proliferation stimulated by different regimens}

The proliferation of splenic T-lymphocytes from immunized mice was determined by the WST-1 cell proliferation assay. Figure 3 shows the SI values determined for splenic Tlymphocytes stimulated by HIV epitope peptides (Figure $3 \mathrm{~A}$ ), or the positive control ConA (Figure 3B). As shown in Figure $3 \mathrm{~A}$, the stimulation of T-lymphocytes by HIV epitope peptides resulted in significant differences in $S I$ values for splenocytes from the rddVTT $_{\text {-CCMp24-only vaccine }}$ group (Group 2) and the rDNA/virus prime-boost group (Group 3) when compared with the PBS control group $(P<0.01)$. No significant difference in $S I$ values was observed between the rDNA-rDNA group (Group 1) and the PBS control group. These results indicate that both rVV-rVV and rDNA-rVV could stimulate a T-lymphocyte specific proliferative response to HIV peptides.

\subsection{Flow cytometric analysis of splenocytes}

The proportion of $\mathrm{CD}^{+}$and $\mathrm{CD}^{+} \mathrm{T}$ cells measured for each experimental group are presented in Figure 4. The DNA/virus prime-boost group (Group 3) and the rddVTT $_{\text {-CCMp24-only vaccine group (Group } 2 \text { ) had higher pro- }}$ portions of $\mathrm{CD}^{+} \mathrm{CD}^{+}(P<0.05)$ and $\mathrm{CD}^{+}{ }^{+} \mathrm{CD} 8^{+}(P<0.01) \mathrm{T}$ cells $/ 10^{6}$ splenocytes than the corresponding PBS control group. No significant difference between Group 2 and Group 3 was observed. However, the percentage of $\mathrm{CD}^{+} \mathrm{T}$ cells for the DNA/virus prime-boost group (Group 3) was higher than for both the pCCMp24-only vaccine group (Group 1) $(P<0.01)$. In addition, the percentage of $\mathrm{CD}^{+} \mathrm{T}$ cells for the rddVTT $_{\text {-CCMp24-only vaccine group was higher }}$ than for the corresponding pCCMp24-only vaccine group $(P<0.05)$.

\subsection{Increased IFN- $\gamma$ secretion following prime-boost immunization and immunologic memory}

To further evaluate the pCCMp24 and $\operatorname{rddVTT}_{- \text {CCMp}_{2} 4}$ prime-boost immunization strategy, splenocytes from mice immunized with pCCMp24- or rddVTT-_CMp24-only vaccine, pCCMp24-rddVTT_-CCMp24 prime-boost, and PBS control were isolated and assessed. The $\mathrm{T}$ cell responses were measured $30 \mathrm{~d}$ after the first immunization $(10 \mathrm{~d}$ post-boost immunization) and at $90 \mathrm{~d}$ post-priming, by IFN- $\gamma$ capture ELISPOT assay against inactivated rddVTT $_{\text {-CCMp24 }}$ and HIV epitope peptides (Figures 5 and 6). At $30 \mathrm{~d}$ post-prime immunization, and following stimulation with inactivated

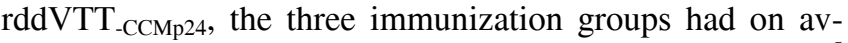
erage greater numbers of IFN- $\gamma$-positive spots (SFC) $/ 10^{5}$ splenocytes than did the PBS control group $(P<0.01)$. This indicated that the immune responses were significantly enhanced for all immunization groups when compared with the PBS control group (Figures 5A and 6). The rdd-

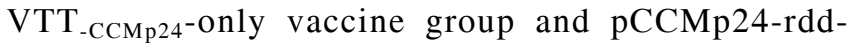
VTT $_{\text {-CCMp24 }}$ prime-boost group had considerably greater numbers of IFN- $\gamma$-positive spots (SFC) $/ 10^{5}$ splenocytes than was observed for the pCCMp24-only vaccine group $(P<0.01)$. No significant differences were detected between Group 2 and Group 3 (Figures 5A and 6). After stimulation

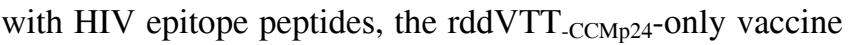
group and pCCMp24-rddVTT-_CMp24 prime-boost group had greater numbers of $\mathrm{SFC} / 10^{5}$ splenocytes than both the pCCMp24-only and PBS control group $(P<0.01$; Figures 5B and 6). The pCCMp24-rddVTT -CMp24 $_{\text {prime-boost group }}$ had greater numbers of SFC/ $10^{5}$ splenocytes than did the rddVTT $_{\text {-CCMp24-only vaccine group }}(P<0.01$; Figures 5B and 

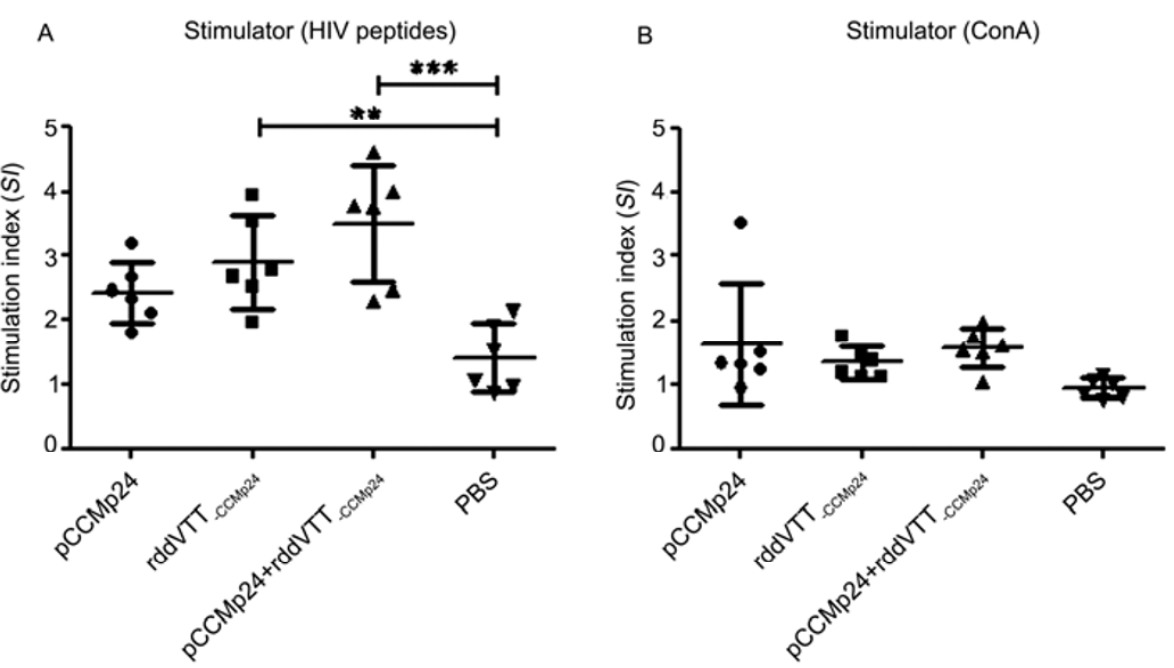

Figure 3 Proliferative analysis of splenocyte from mice immunized with pCCMp24, rddVTT-CCMp24, VTT, or PBS at $10 \mathrm{~d}$ post-immunization. Approximately $2.5 \times 10^{5}$ splenocytes were stimulated with HIV epitope peptides or the positive control ConA. The stimulation index indicates the proliferative capacity of splenocytes from each group when stimulated with different agents. The data represents the mean $(n=6) \pm$ SD. Asterisks represent statistically significant differences; **, $P<0.01 ; * * *, P<0.001$.
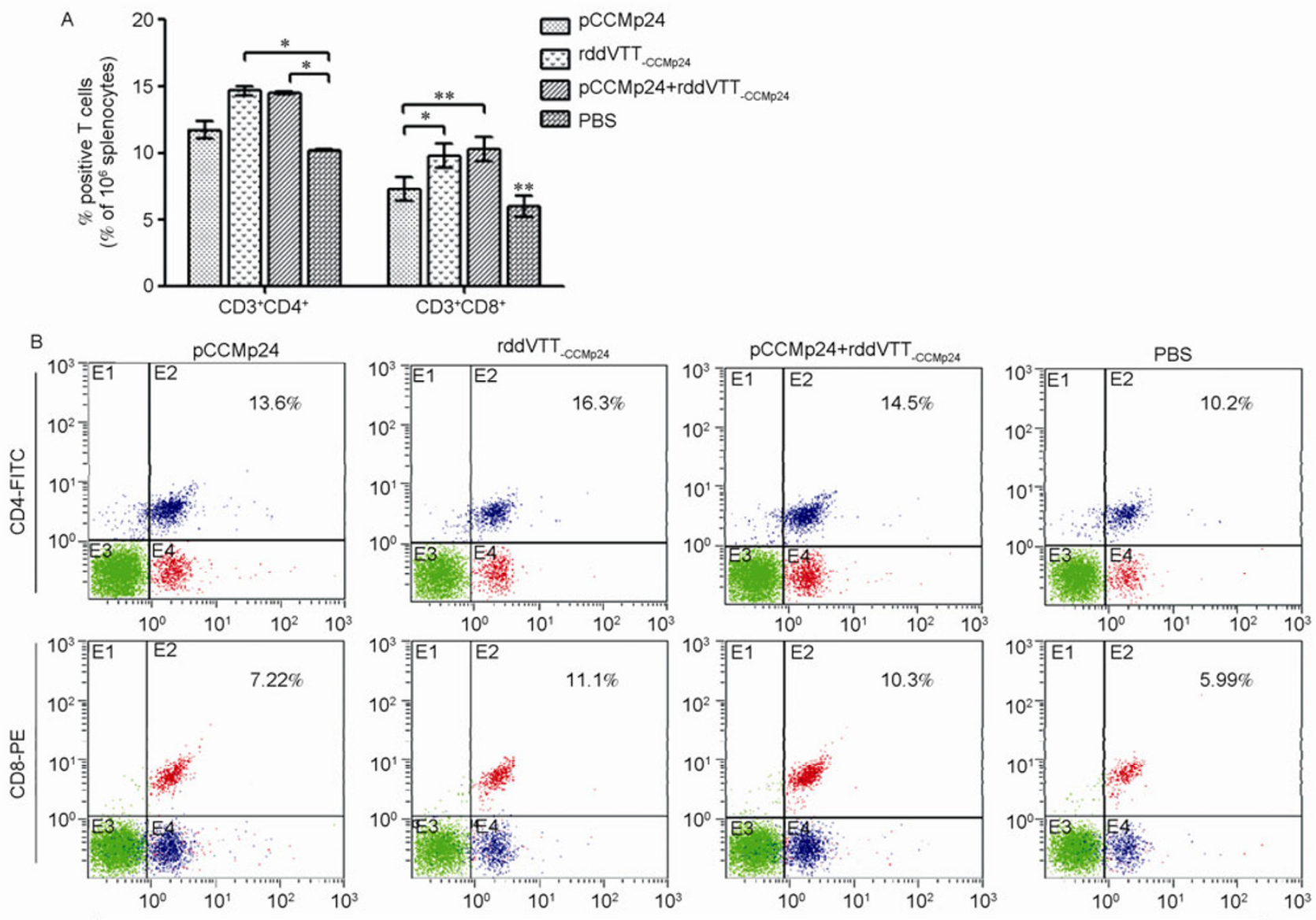

CD3-PerCP/Cy5.5

Figure 4 Phenotypic analysis of $\mathrm{CD}^{+}$and $\mathrm{CD}^{+} \mathrm{T}$ cells performed with flow cytometry. Splenocytes obtained at $10 \mathrm{~d}$ post-immunization from mice immunized with rddVTT.CCMp24 or VTT were used to analyze the percentages of $\mathrm{CD}^{+} \mathrm{CD}^{+}$and $\mathrm{CD}^{+} \mathrm{CD}^{+} \mathrm{T}$ cells using flow cytometry. A, Bar graph presenting the percentages of $\mathrm{CD}_{3}{ }^{+} \mathrm{CD} 4^{+}$and $\mathrm{CD}^{+} \mathrm{CD}^{+} \mathrm{T}$ cells per $10^{6}$ splenocytes (mean $\pm \mathrm{SD}$ ). Asterisks represent significant differences; $*, P<0.05 ; * *$, $P<0.01$. B, Representative scatterplots depicting cell surface antigen staining. The percentages of $\mathrm{CD}^{+} \mathrm{CD} 4^{+}$and $\mathrm{CD} 3^{+} \mathrm{CD} 8^{+} \mathrm{T}$ cells per $10^{6}$ splenocytes are indicated in the upper right corner. 

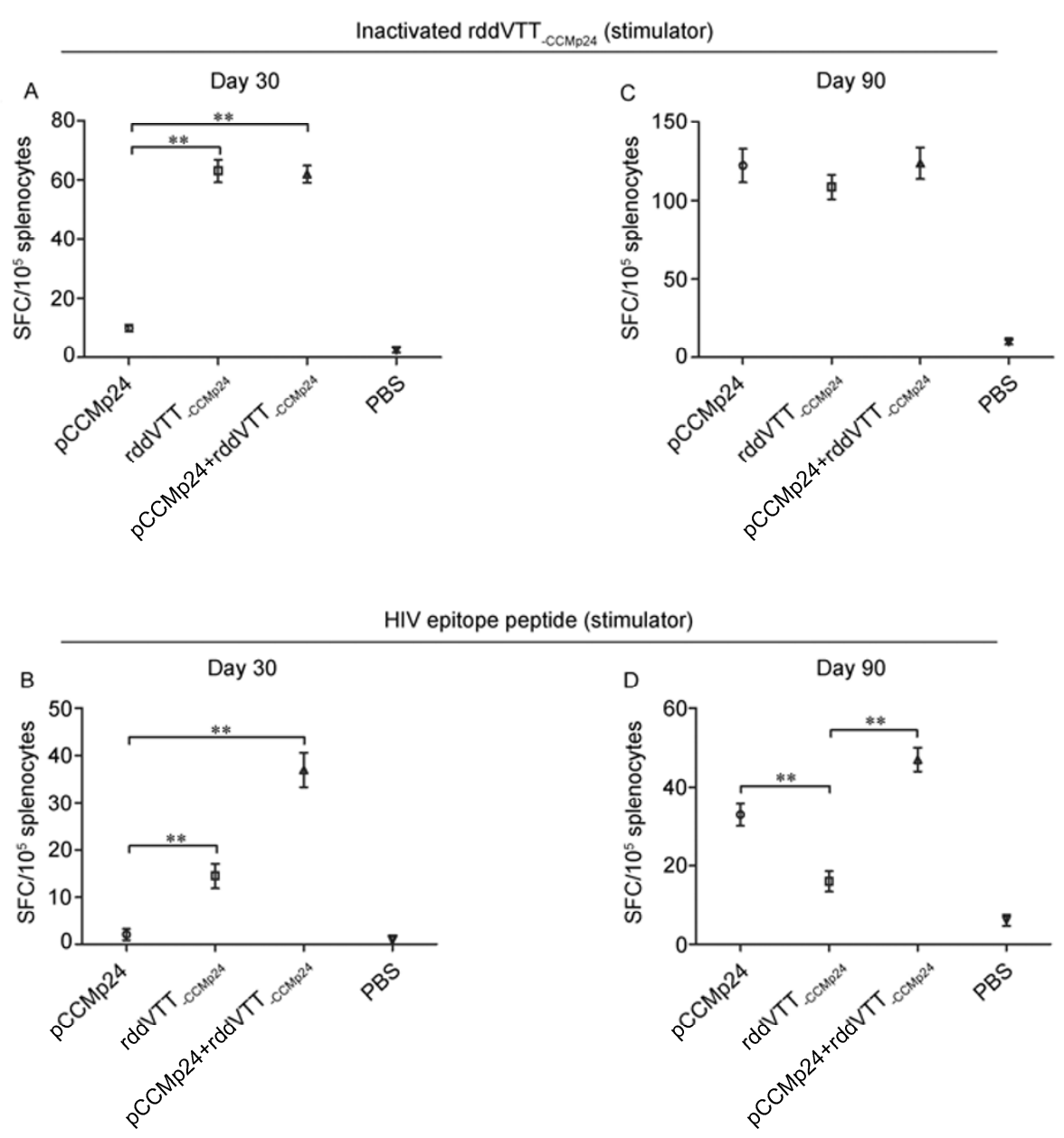

Figure 5 T cell IFN- $\gamma$ secretion measured by ELISPOT. About $1 \times 10^{5}$ splenocytes from mice immunized with pCCMp24, rddVTT -CCMp24, VTT, or PBS at $30 \mathrm{~d}(\mathrm{~A}$ and $\mathrm{B})$ and $90 \mathrm{~d}(\mathrm{C}$ and $\mathrm{D})$ after prime immunization were stimulated with inactivated rddVTT CCMp24 $(\mathrm{A}$ and $\mathrm{C})$ and HIV CTL epitope peptides (B and D). In each panel, the data represent the mean $(n=6) \pm \mathrm{SD}$. Asterisks represent statistically significant differences; $* *, P<0.01$.

6). These results indicate that the prime-boost immunization strategy could induce greater specific cellular immune responses than single-shot vaccinations against HIV epitopes.

At $90 \mathrm{~d}$ post-prime immunization $(60 \mathrm{~d}$ post boost immunization), IFN- $\gamma$-positive spots (SFC) $/ 10^{5}$ splenocytes were measured for evaluating cellular immunologic memory. The average number of IFN- $\gamma$-positive spots $/ 10^{5}$ splenocytes for all immunization groups was greater against

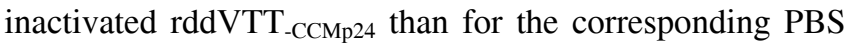
control group $(P<0.01$; Figure $5 \mathrm{C})$. The pCCMp24rddVTT $_{- \text {CCMp24 }}$ prime-boost group had greater numbers of $\mathrm{SFC} / 10^{5}$ splenocytes than did the pCCMp24-only group

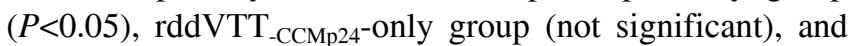
PBS group $(P<0.01)$ against HIV epitopes (Figures 5D and $6)$. Interestingly, the pCCMp24-only group had higher average numbers of SFC $/ 10^{5}$ splenocytes than the ddVTT $_{- \text {CCMp24- }^{-}}$ only group against HIV epitopes $(P<0.01$; Figure 5D). The-


following pCCMp24 priming could induce HIV-specific cellular immune responses and immunologic memory.

\section{Discussion}

Vaccination has greatly impacted the health of human and animals in the past. To date, vaccination still plays a crucial role in preventing and controlling infectious diseases and cancers. With regard to the development of an effective AIDS vaccine, a myriad of problems and challenges have been encountered by researchers across a number studies. Such challenges include using appropriate animal models for vaccine evaluation, and effective immunogen design and immunization strategies $[19,39,40]$. VAX003 and VAX004 based on recombinant gp120 could not delay disease progression in phase III human clinical trials, and the antibodies were unable to neutralize most HIV-1 strains [8,41]. Considering the high genetic variability of HIV, it seems unlikely that a single antigen would prove efficacious in protecting against HIV infection and disease progression. Therefore, antigen multimers, multi-epitope, or a combination of various antigens could be used to address this issue and to elicit a strong immune response against HIV [13,15]. 


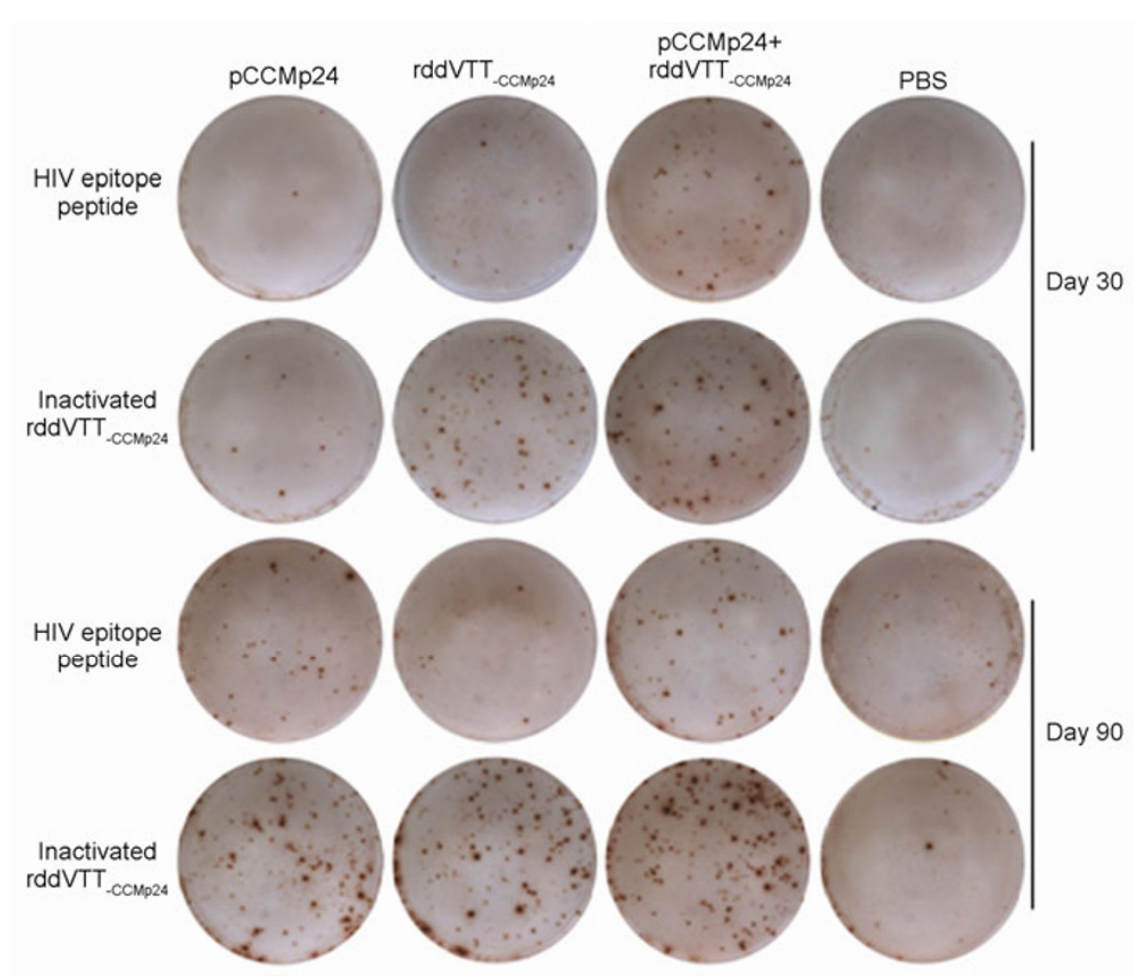

Figure 6 Representative IFN- $\gamma$ spot forming cells under the stimulation of HIV epitope peptides and inactivated rddVTT -CCMp24 $_{\text {are }}$ shown. The splenocytes were isolated from mice immunized with pCCMp24, rddVTT-CCMp24, VTT, or PBS at 30 and 90 d post-prime immunization. Each spot represents one T lymphocyte-secreting IFN- $\gamma$.

In previous study, 29 predominant epitopes of HIV including 23 epitopes of HIV-1, three epitopes of HIV-2, a Th cell universal epitope, a B cell universal epitope, and a tetanus toxin epitope were selected to synthesize and form a fusion gene MEGN. The p24 protein of HIV-1 was inserted into the multi-epitopes, of the aforementioned fusion gene, and named MEGNp24. To enhance the immunogenicity of the immunogen, a CpG ODN motif and CTB sequence were included upstream of MEGNp24, and the antigen designated CCMp24.

Recombinant DNA vaccines and live recombinant virusbased vaccines are the focus of many studies. Recent studies have documented the use of a number of different vaccines including viral vector vaccines or recombinant proteins as a boost following priming in an attempt to enhance the immune response induced by a single vaccine. DNA priming followed by MVA boosting, and vice versa, may provide better protection against malaria infection in mice compared with using the DNA or MVA vector alone [42]. In a previous study, different administration sequences with recombinant DNA and recombinant poxvirus were investigated in our laboratory. In the present study, virus vector booster immunization following priming with DNA was applied. Although researchers do not completely understand the principle behind the enhanced immune response for prime-boost strategies or the reason why priming with the recombinant DNA is followed by another modality such as viral vector vaccine or recombinant protein, a number of studies have documented the use of DNA/live viral recombinant in immunization regimens [43]. Several studies in non-human primates have demonstrated a significant improvement in immunogenicity for a single DNA vaccine when a viral vector vaccine was administered as a boost following priming [26]. Such synergy between DNA and viral vector vaccines was observed in non-human primates when used for boosting with poxviruses such as vaccinia virus $[9,33,34,44,45]$, Ad5 $[46,47]$ or vesicular stomatitis virus [48].

Two attenuated vaccinia virus strains, MVA and NYVAC, have been used as vectors in vaccine research $[3,49,50]$. MVA-based therapeutic vaccines have been used in the treatment of some major chronic infections, including viral hepatitis, AIDS, and tuberculosis. Phase 1 and 2 clinical trials for MVA-based therapeutic vaccines have started to show significant results and promise for the effective treatment of chronic infectious diseases [51]. In rhesus macaques, DNA-MVA vaccine schedules expressing SIVmac$239 \mathrm{gag}, \mathrm{pr}, \mathrm{rt}$, and $e n v$ genes, elicited high numbers of $\mathrm{CD}^{+}$and $\mathrm{CD}^{+} \mathrm{T}$ cells, and high titers and avidity for Env-specific IgG [16]. In a recent phase I clinical trial (RISVAC02), an MVA-based HIV vaccine expressing the env, gag, pol, and nef proteins of HIV-1 subtype B (MVA-B) was safe, well tolerated, and elicited strong and durable T-cell and antibody responses in $75 \%$ and $95 \%$ of volunteers [52]. The recombinant vaccinia virus vaccine rddVTT $_{\text {-CCMp24 }}$ used in this study was constructed previously 
and based on an attenuated vaccinia virus Tian Tan (deletion of $T E 3 L$ and $T K$ genes).

In the present study, the immunogenicity of various immunization strategies using pCCMp24 and rddVTT-CCMp24 expressing CTB, HIV multi-epitopes, and p24 protein (CCMp24) was investigated in mice. Booster administration


erated strong humoral and $\mathrm{T}$ cell responses, particularly IFN- $\gamma$-mediated cellular immune responses against HIV peptides. The prime-boost immunization schedule can induced more $\mathrm{CD}^{+} \mathrm{T}$ cells than vaccination with DNA

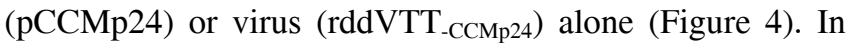


sary following DNA priming to enhance IFN- $\gamma$-mediated cellular immune responses upon stimulation by HIV peptide pools compared with single-shot DNA/DNA or virus/virus (Figure 5B and D). There was no significant difference in the levels of cytokines (IL-2 and IL-4) in peripheral blood for the three different immunization schedules (Figure 2). With regard to HIV-specific antibody, rddVTT-СCMp24 booster immunization following DNA priming induced antibody production more rapidly than for the DNA booster, and the antibody levels showed no significant difference compared with virus/virus immunization (Figure 1C). These data may serve as a basis for future pre-clinical studies of recombinant DNA/vaccinia virus immunization schedules.

We thank Liu YanYu and Li Ping for their technical support during the animal studies. This work was supported by the National Natural Science Foundation of China (81001342), the National Basic Research Program of China (2011CB512110), and the National Mega Project on Major Infectious Diseases Prevention (2012ZX10001005-006).

1 Garcia-Arriaza J, Najera J L, Gomez C E, et al. A candidate HIV/AIDS vaccine (MVA-B) lacking vaccinia virus gene C6L enhances memory HIV-1-specific T-cell responses. PLoS ONE, 2011, 6: e24244

2 Gomez C E, Perdiguero B, Jimenez V, et al. Systems analysis of MVA-C induced immune response reveals its significance as a vaccine candidate against HIV/AIDS of clade C. PLoS ONE, 2012, 7: e35485

3 Kibler K V, Gomez C E, Perdiguero B, et al. Improved NYVAC-based vaccine vectors. PLoS ONE, 2011, 6: e25674

4 Hemachandra A, Puls R L, Sirivichayakul S, et al. An HIV-1 clade A/E DNA prime, recombinant fowlpox virus boost vaccine is safe, but non-immunogenic in a randomized phase I/IIa trial in Thai volunteers at low risk of HIV infection. Hum Vaccin, 2010, 6: $835-840$

5 Song $\mathrm{Y}$, Zhang $\mathrm{L} \mathrm{S}$, Wang $\mathrm{H}$, et al. Immune responses of mice to prime-boost vaccination with the recombinant DNA and Fowlpox virus both expressing HIV-2 Gag-gp105. Acta Virol, 2010, 54: 293-296

6 Shiver J W, Fu T M, Chen L, et al. Replication-incompetent adenoviral vaccine vector elicits effective anti-immunodeficiencyvirus immunity. Nature, 2002, 415: 331-335

7 D'Souza M P, Frahm N. Adenovirus 5 serotype vector-specific immunity and HIV-1 infection: a tale of T cells and antibodies. AIDS, 2010, 24: 803-809

8 Pitisuttithum P, Gilbert P, Gurwith M, et al. Randomized, doubleblind, placebo-controlled efficacy trial of a bivalent recombinant glycoprotein 120 HIV-1 vaccine among injection drug users in Bangkok, Thailand. J Infect Dis, 2006, 194: 1661-1671

9 Cox J H, Ferrari M G, Earl P, et al. Inclusion of a CRF01_AE HIV envelope protein boost with a DNA/MVA prime-boost vaccine: Impact on humoral and cellular immunogenicity and viral load reduction after SHIV-E challenge. Vaccine, 2012, 30: 1830-1840

10 Ferrantelli F, Maggiorella M T, Schiavoni I, et al. A combination HIV vaccine based on Tat and Env proteins was immunogenic and protected macaques from mucosal SHIV challenge in a pilot study. Vaccine, 2011, 29: 2918-2932

11 Sealy R, Slobod K S, Flynn P, et al. Preclinical and clinical development of a multi-envelope, DNA-virus-protein (D-V-P) HIV-1 vaccine. Int Rev Immunol, 2009, 28: 49-68

12 Li C, Shen Z, Li X, et al. Protection against SHIV-KB9 infection by combining rDNA and rFPV vaccines based on HIV multiepitope and p24 protein in Chinese rhesus macaques. Clin Dev Immunol, 2012, 2012: 958404

13 Sundaramurthi J C, Swaminathan S, Hanna L E. Resistanceassociated epitopes of HIV-1C-highly probable candidates for a multi-epitope vaccine. Immunogenetics, 2012, 64: 767-772

14 Kovacs J M, Nkolola J P, Peng H, et al. HIV-1 envelope trimer elicits more potent neutralizing antibody responses than monomeric gp120. Proc Natl Acad Sci USA, 2012, 109: 12111-12116

15 Stephenson K E, Sanmiguel A, Simmons N L, et al. Full-length HIV-1 immunogens induce greater magnitude and comparable breadth of $\mathrm{T}$ lymphocyte responses to conserved HIV-1 regions compared with conserved-region-only HIV-1 immunogens in rhesus monkeys. J Virol, 2012, 86: 11434-11440

16 Lai L, Kwa S F, Kozlowski P A, et al. SIVmac239 MVA vaccine with and without a DNA prime, similar prevention of infection by a repeated dose SIVsmE660 challenge despite different immune responses. Vaccine, 2012, 30: 1737-1745

17 Goepfert P A, Elizaga M L, Sato A, et al. Phase 1 safety and immunogenicity testing of DNA and recombinant modified vaccinia Ankara vaccines expressing HIV-1 virus-like particles. J Infect Dis, 2011, 203: 610-619

18 IAVIReport-The publication on AIDS vaccine research. http://www. iavireport.org, August 13, 2012

19 Saunders K O, Rudicell R S, Nabel G J. The design and evaluation of HIV-1 vaccines. AIDS, 2012, 26: 1293-1302

20 Rerks-Ngarm S, Pitisuttithum P, Nitayaphan S, et al. Vaccination with ALVAC and AIDSVAX to prevent HIV-1 infection in Thailand. N Engl J Med, 2009, 361: 2209-2220

21 van Drunen Littel-van den Hurk S, Hannaman D. Electroporation for DNA immunization: clinical application. Expert Rev Vaccines, 2010, 9: 503-517

22 Graham B S, Koup R A, Roederer M, et al. Phase 1 safety and immunogenicity evaluation of a multiclade HIV-1 DNA candidate vaccine. J Infect Dis, 2006, 194: 1650-1660

23 Schadeck E B, Sidhu M, Egan M A, et al. A dose sparing effect by plasmid encoded IL-12 adjuvant on a SIVgag-plasmid DNA vaccine in rhesus macaques. Vaccine, 2006, 24: 4677-4687

24 Ganguly S, Liu J, Pillai V B, et al. Adjuvantive effects of anti-4-1BB agonist $\mathrm{Ab}$ and 4-1BBL DNA for a HIV-1 Gag DNA vaccine: different effects on cellular and humoral immunity. Vaccine, 2010, 28: 1300-1309

25 Spearman P, Kalams S, Elizaga M, et al. Safety and immunogenicity of a CTL multiepitope peptide vaccine for HIV with or without GM-CSF in a phase I trial. Vaccine, 2009, 27: 243-249

26 Girard M P, Osmanov S, Assossou O M, et al. Human immunodeficiency virus (HIV) immunopathogenesis and vaccine development: a review. Vaccine, 2011, 29: 6191-6218

27 Oxenius A, Martinic M M, Hengartner $\mathrm{H}$, et al. CpG-containing oligonucleotides are efficient adjuvants for induction of protective antiviral immune responses with T-cell peptide vaccines. J Virol, 1999, 73: 4120-4126

28 Gallichan W S, Woolstencroft R N, Guarasci T, et al. Intranasal immunization with $\mathrm{CpG}$ oligodeoxynucleotides as an adjuvant dramatically increases $\operatorname{Ig} \mathrm{A}$ and protection against herpes simplex 
virus-2 in the genital tract. J Immunol, 2001, 166: 3451-3457

29 Belyakov I M, Isakov D, Zhu Q, et al. Enhancement of $\mathrm{CD}^{+} \mathrm{T}$ cell immunity in the lung by $\mathrm{CpG}$ oligodeoxynucleotides increases protective efficacy of a modified vaccinia Ankara vaccine against lethal poxvirus infection even in a CD4-deficient host. J Immunol, 2006, 177: 6336-6343

30 Olvera-Gomez I, Hamilton S E, Xiao Z, et al. Cholera toxin activates nonconventional adjuvant pathways that induce protective CD8 T-cell responses after epicutaneous vaccination. Proc Natl Acad Sci USA, 2012, 109: 2072-2077

31 Elena Gomez C, Perdiguero B, Garcia-Arriaza J, et al. Poxvirus vectors as HIV/AIDS vaccines in humans. Hum Vaccin Immunother, 2012, 8: 1192-1207

32 Makitalo B, Lundholm P, Hinkula J, et al. Enhanced cellular immunity and systemic control of SHIV infection by combined parenteral and mucosal administration of a DNA prime MVA boost vaccine regimen. J Gen Virol, 2004, 85: 2407-2419

33 Bertley F M, Kozlowski P A, Wang S W, et al. Control of simian/ human immunodeficiency virus viremia and disease progression after IL-2-augmented DNA-modified vaccinia virus Ankara nasal vaccination in nonhuman primates. J Immunol, 2004, 172: 37453757

34 Manrique M, Micewicz E, Kozlowski P A, et al. DNA-MVA vaccine protection after $\mathrm{X} 4 \mathrm{SHIV}$ challenge in macaques correlates with day-of-challenge antiviral $\mathrm{CD}^{+}$cell-mediated immunity levels and postchallenge preservation of CD4+ T cell memory. AIDS Res Hum Retroviruses, 2008, 24: 505-519

35 Sandstrom E, Nilsson C, Hejdeman B, et al. Broad immunogenicity of a multigene, multiclade HIV-1 DNA vaccine boosted with heterologous HIV-1 recombinant modified vaccinia virus Ankara. J Infect Dis, 2008, 198: 1482-1490

36 Bakari M, Aboud S, Nilsson C, et al. Broad and potent immune responses to a low dose intradermal HIV-1 DNA boosted with HIV-1 recombinant MVA among healthy adults in Tanzania. Vaccine, 2011, 29: $8417-8428$

37 Gorse G J, Newman M J, deCamp A, et al. DNA and modified vaccinia virus Ankara vaccines encoding multiple cytotoxic and helper T-lymphocyte epitopes of human immunodeficiency virus type 1 (HIV-1) are safe but weakly immunogenic in HIV-1uninfected, vaccinia virus-naive adults. Clin Vaccine Immunol, 2012, 19: 649-658

38 Shen Z W, Jin H T, Li C, et al. Adjuvant effects of dual costimulatory molecules on cellular responses to HIV multipleepitope DNA vaccination. Chem Res Chin Univ, 2009, 25: 347-352

39 McBurney S P, Ross T M. Viral sequence diversity: challenges for AIDS vaccine designs. Expert Rev Vaccines, 2008, 7: 1405-1417
40 Hatziioannou T, Ambrose Z, Chung N P, et al. A macaque model of HIV-1 infection. Proc Natl Acad Sci USA, 2009, 106: 4425-4429

41 Flynn N M, Forthal D N, Harro C D, et al. Placebo-controlled phase 3 trial of a recombinant glycoprotein 120 vaccine to prevent HIV-1 infection. J Infect Dis, 2005, 191: 654-665

42 Schneider J, Gilbert S C, Blanchard T J, et al. Enhanced immunogenicity for $\mathrm{CD}^{+} \mathrm{T}$ cell induction and complete protective efficacy of malaria DNA vaccination by boosting with modified vaccinia virus Ankara. Nat Med, 1998, 4: 397-402

43 Liu M A. Immunologic basis of vaccine vectors. Immunity, 2010, 33: 504-515

44 Liu L, Hao Y, Luo Z, et al. Broad HIV-1 neutralizing antibody response induced by heterologous gp140/gp145 DNA prime-vaccinia boost immunization. Vaccine, 2012, 30: 4135-4143

45 Hel Z, Nacsa J, Tryniszewska E, et al. Containment of simian immunodeficiency virus infection in vaccinated macaques: correlation with the magnitude of virus-specific pre- and postchallenge $\mathrm{CD}^{+}$and $\mathrm{CD}^{+} \mathrm{T}$ cell responses. J Immunol, 2002, 169: 4778-4787

46 Mascola J R, Sambor A, Beaudry K, et al. Neutralizing antibodies elicited by immunization of monkeys with DNA plasmids and recombinant adenoviral vectors expressing human immunodeficiency virus type 1 proteins. J Virol, 2005, 79: 771-779

47 Koup R A, Roederer M, Lamoreaux L, et al. Priming immunization with DNA augments immunogenicity of recombinant adenoviral vectors for both HIV-1 specific antibody and T-cell responses. PLoS ONE, 2010, 5: e9015

48 Schell J, Rose N F, Fazo N, et al. Long-term vaccine protection from AIDS and clearance of viral DNA following SHIV89.6P challenge. Vaccine, 2009, 27: 979-986

49 Shephard E, Burgers W A, Van Harmelen J H, et al. A multigene HIV type 1 subtype $\mathrm{C}$ modified vaccinia Ankara (MVA) vaccine efficiently boosts immune responses to a DNA vaccine in mice. AIDS Res Hum Retroviruses, 2008, 24: 207-217

50 Esteban M. Attenuated poxvirus vectors MVA and NYVAC as promising vaccine candidates against HIV/AIDS. Hum Vaccin, 2009, 5: $867-871$

51 Boukhebza H, Bellon N, Limacher J M, et al. Therapeutic vaccination to treat chronic infectious diseases: current clinical developments using MVA-based vaccines. Hum Vaccin Immunother, 2012, 8

52 Garcia F, Bernaldo de Quiros J C, Gomez C E, et al. Safety and immunogenicity of a modified pox vector-based HIV/AIDS vaccine candidate expressing Env, Gag, Pol and Nef proteins of HIV-1 subtype B (MVA-B) in healthy HIV-1-uninfected volunteers: a phase I clinical trial (RISVAC02). Vaccine, 2011, 29: 8309-8316

Open Access This article is distributed under the terms of the Creative Commons Attribution License which permits any use, distribution, and reproduction in any medium, provided the original author(s) and source are credited. 